\title{
A ideia por trás do Colóquio Brasileiro de Morfologia (CBM) e os trabalhos do III CBM publicados neste volume
}

The idea behind the Brazilian Colloquium of Morphology and the papers of the III CBM published in this volume

\author{
Ana Paula Scher \\ (Universidade de São Paulo, São Paulo, São Paulo, Brasil) \\ Indaiá de Santana Bassani \\ (Universidade Federal de São Paulo, Guarulhos, São Paulo, Brasil) \\ Paula Roberta Gabbai Armelin \\ (Universidade Federal de Juiz de Fora, Juiz de Fora, Minas Gerais, Brasil)
}

\section{RESUMO:}

Este artigo configura-se como uma introdução ao conteúdo que o segue, tendo dois objetivos gerais: (i) apresentar brevemente o histórico do Colóquio Brasileiro de Morfologia, CBM, e (ii) oferecer um panorama dos artigos que compõem este volume da Revista D.E.L.T.A.,. O CBM configurase como um importante evento no cenário nacional, uma vez que supre uma lacuna relevante no cenário linguístico brasileiro, ao colocar no centro das discussões aspectos morfológicos das línguas naturais. A morfologia, sendo naturalmente uma área de interface, com fenômenos sintáticos, fonológicos e semânticos, tem o potencial de contribuir substantivamente para a construção de teorias linguísticas interessantes. Os artigos aceitos para publicação neste volume da Revista D.E.L.T.A., que reúne trabalhos apresentados no III CBM, são evidência para essa afirmação.

Palavras-chave: Morfologia. Morfossintaxe. Morfofonologia. Morfossemântica. 


\begin{abstract}
:
This paper is an introduction to the content that follows it, having two general goals: (i) to briefly present the history of the Brazilian Colloquium of Morphology, CBM, and (ii) to offer an overview of the papers in this volume. The CBM is an important event on the national scene, as it fills a relevant gap in the Brazilian linguistic scenario by placing morphological aspects of natural languages at the center of discussions. Morphology, being naturally an interface area, with syntactic, phonological and semantic phenomena, has the potential to contribute substantively to the construction of interesting linguistic theories. The articles accepted for publication in this volume of D.E.L.T.A., which brings together works presented in III CBM, are evidence for this claim.
\end{abstract}

Keywords: Morphology; Morphosyntax. Morphophonology. Morphossemantics.

\title{
1. Uma breve retomada histórica do CBM
}

Durante as atividades do VII Congresso Internacional da ABRALIN e do XX Instituto da ABRALIN, realizados em Curitiba, em fevereiro de 2011, o Luiz Carlos Schwindt e nós do GREMD - Grupo de Estudos em Morfologia Distribuída da USP - tivemos a ideia de reunir nossos grupos de pesquisa em um evento informal em que nossos trabalhos e os de nossos orientandos pudessem ser apresentados e discutidos. Ficou decidido que faríamos um encontro em Porto Alegre, na UFRGS, numa realização conjunta da UFRGS e da USP.

A ideia tomou corpo e foi concretizada, sob a coordenação do Luiz Carlos, na forma do I Colóquio Brasileiro de Morfologia, o I CBM, que reuniu pesquisadores e estudantes dessa área, contemplando, ainda, estudos nas suas interfaces (fonológica, sintática e semântico-pragmática) em diferentes concepções teóricas e descritivas. A ideia era compartilhar os achados dessas investigações e contribuir, assim, para a consolidação da área de Morfologia no Brasil. Em sua primeira versão, o evento contou com a participação de palestrantes como Margarida Basílio (PUC-Rio), Leda Bisol (PUC-RS), Carlos Alexandre Gonçalves (UFRJ), Sergio de Moura Menuzzi (UFRGS), Ana Paula Scher (USP), Luiz Carlos Schwindt (UFRGS), Paulo Chagas de Souza (USP) e Seung Hwa-Lee (UFMG).

A segunda versão do CBM, o II Colóquio Brasileiro de Morfologia, aconteceu em julho de 2013, na UFRJ, Rio de Janeiro, e homenageou a Professora Margarida Basílio. O evento foi organizado pelos professores Carlos Alexandre V. Gonçalves e Maria Lúcia Leitão de Almeida e contou com a participação dos 
seguintes palestrantes de universidades portuguesas e brasileiras: Ieda Maria Alves (USP), Hanna Batoreo (UAB, Portugal), Margarita Correia (ILTEC, Portugal), Miriam Lemle (UFRJ), José Borges Neto (UFPR), Graça Maria Rio-Torto (Univ. Coimbra, Portugal), Ana Paula Scher (USP), Maria Cristina Figueiredo Silva (UFPR), Luiz Carlos de Assis Rocha (UFMG), Maria Carlota Rosa (UFRJ), Mario Eduardo Viaro (USP), Alina Villalva (ULisboa, Portugal) e Seung Hwa-Lee (UFMG).

$\mathrm{Na}$ terceira edição do CBM, continuamos a tradição, já estabelecida nas versões anteriores desse evento, de oferecer um espaço de discussão sobre diferentes fenômenos e de acordo com diversas abordagens morfológicas. A alta qualidade das pesquisas realizadas nesse campo atualmente, no Brasil, enriqueceu o III Colóquio Brasileiro de Morfologia, trouxe ganhos para seus participantes e contribuiu para o avanço da investigação em Morfologia e suas interfaces no país. Os resultados dessas investigações, feitas dentro das diversas e complementares linhas do fazer morfológico no Brasil, indicaram um futuro frutífero para a área. Além disso, indicaram, também, a pertinência de eventos especializados, como o CBM, em que pesquisadores com interesses comuns podem se beneficiar do olhar e dos comentários de seus pares sobre suas pesquisas. Esses fatos nos alegraram e confirmaram o caráter do Colóquio Brasileiro de Morfologia como um espaço qualificado de discussão científica.

O III CBM foi realizado em novembro de 2015, numa promoção do Departamento de Linguística da USP. Embora tenha se realizado nas dependências da USP, a organização do III CBM contou com a colaboração dos professores Rafael Dias Minussie Indaiá de Santana Bassani, da UNIFESP, Aline Garcia Rodero Takahira e Paula Roberta Gabbai Armelin, da UFJF, João Paulo Lazzarini Cyrino, da UFBA, e Julio William Curvelo Barbosa, da UNESPAR - Campus Paranaguá. Além desses colegas, a Comissão Organizadora desse evento contou, ainda, com a participação dos então doutorandos do Programa de Pós-graduação em Linguística da USP, Janayna Maria da Rocha Carvalho e Vitor Augusto Nóbrega, e, também, dos então mestrandos do mesmo Programa, Klauber Renan Dutra de Oliveira e Mayara Espadaro. Todos os participantes da Comissão Organizadora são ou foram membros do GREMD, que comemorava os 11 anos de sua formação nessa universidade. O III CBM foi, portanto, uma realização conjunta de, pelo menos, cinco universidades brasileiras e contou com a participação de representantes de várias outras universidades do país: os palestrantes convidados eram Luiz Carlos Schwindt (UFRGS) e Maria Cristina Figueiredo-Silva (UFPR), e os apresentadores de comunicações também representavam diferentes instituições de ensino superior no Brasil. Além disso, em comemoração ao $11^{\circ}$ aniversário do GREMD, foi organizado um debate em que os trabalhos desenvolvidos por membros desse grupo foram apresentados e discutidos pelos seguintes colegas pesquisadores convidados: 
Danniel Carvalho (UFBA), Alessandro Boechat de Medeiros (UFRJ), Filomena Sândalo (UNICAMP) e Luciana Storto (USP).

Foram momentos de intenso debate acadêmico-científico, uma boa parte dos quais fica registrada com a publicação deste volume. A produtividade dos grupos de pesquisa em Morfologia no Brasil continua e se mantém crescente. Neste momento, dá-se a preparação do IV CBM, previsto para se realizar em Salvador, em março de 2018, numa promoção da UFBA. Bons frutos estão sendo colhidos daquela semente plantada na ABRALIN de 2011!

Prova disso são os artigos aceitos para publicação neste volume da Revista D.E.L.T.A., que reúne trabalhos apresentados no III CBM.

\section{Sobre os artigos contidos neste volume: as diversas interfa- ces da Morfologia}

A pluralidade de temas discutidos nesta edição da revista, unidos pela abordagem direta da Morfologia de uma língua natural, ou pela investigação de suas interfaces fonético-fonológica, sintática, semântico-pragmática, dentre outras, espelha o caráter multifacetado do evento. Assim, os artigos aqui apresentados tratam de uma série de fenômenos empíricos que suscitam questões morfofonológicas, morfossemânticas ou morfossintáticas para as quais são oferecidos tratamentos fundamentados em abordagens descritivas, teóricas e experimentais. Estamos convencidas de que a riqueza dos achados empíricos e teóricos e dos métodos expostos neste volume representa uma ampla contribuição para os interessados em Morfologia.

Nossa discussão tem início com o artigo intitulado Restrições de localidade nas formações de diminutivo do português brasileiro: mapeando uma relação de dependência entre -inh e gênero, de Paula Roberta Gabbai Armelin, que apresenta, a partir de uma perspectiva sintática do processo de formação de palavras (Halle; Marantz 1993, Marantz 1997, Borer 2003, 2005a, 2005b, 2013), uma análise para os diminutivos construídos com o sufixo -inh no português brasileiro (PB). Após mapear evidências empíricas para as relações de localidade que se estabelecem entre tal afixo, a raiz e o núcleo de gênero, a autora propõe que -inh não projeta seu próprio rótulo na estrutura sintática, tendo de integrar uma projeção sintática de gênero, que é considerada parte da projeção estendida dos nominais.

A morfologia de concordância nominal de número vem explorada no artigo intitulado Concordância nominal variável de número e saliência fônica: um estudo experimental, de Cristina Azalim, Mercedes Marcilese, Cristina Name, Lilian Scher e Lydsson Gonçalves. Os autores investigam, por meio de uma metodologia experimental, o papel da saliência fônica na marcação morfológica da concordância nominal em dados do PB. Além de apresentar resultados que 
indicam que saliência fônica deve ser entendida em termos não só de quantidade de material fônico, mas de tonicidade, o artigo representa um exercício de investigação morfológica no contexto de diálogo entre a Psicolinguística e a Sociolinguística Variacionista.

Julio William Curvelo Barbosa, por sua vez, retoma as discussões a respeito da existência de construções resultativas no PB no artigo intitulado Ter estado resultante não é ter construção resultativa: predicados secundários pseudo-resultativos e orações adjuntas de resultado no português brasileiro. $\mathrm{O}$ autor identifica dois grupos distintos de dados entre os que a literatura classifica como resultativas no PB: as construções pseudo-resultativas, cujos predicados secundários são modificadores do estado resultante, e as orações adjuntas de resultado, uma estrutura com dois sintagmas verbais diferentes, com as mesmas propriedades de evento das construções resultativas. Sustentando a hipótese de que resultativas genuínas são estruturas mono-oracionais com um verbo dotado de informações semânticas de modo/maneira e um predicado que denota o estado resultante obtido pela ação do verbo sobre o objeto da sentença, Julio Barbosa propõe, através da noção de emolduração desenvolvida por Talmy $(2000,2009$, 2012) no âmbito da Linguística Cognitiva, não haver qualquer equivalente sintático - e consequentemente semântico - às construções resultativas no PB.

Uma investigação estrutural sobre a interface entre a Morfologia e a Semântica tem lugar no artigo intitulado (Des)construindo relações entre agentividade, causa e morfologia em verbos de mudança de estado do português brasileiro, de Indaiá de Santana Bassani, que aborda questões sobre a decomposição sintática do evento. Usando testes de formação de sentença transitiva com agente, transitiva com causa, intransitiva e passiva, a autora classifica empiricamente e analisa 136 verbos de mudança de estado em seis tipos de eventos (incoativo, causativo, causativo subespecificado para [ag], totalmente subespecificado, causativo agentivo estrito e causativo estrito com voice). Fundamentada pelo modelo da Morfologia Distribuída, a autora analisa tais verbos por meio da decomposição sintática do evento, levando em conta, ao mesmo tempo, a tipologia semântica da raiz, a morfologia verbal e suas possíveis relações com o argumento externo.

No artigo intitulado Sobre a produtividade e a semântica do prefixo -des no português brasileiro atual, de Camila De Bona e Pablo Nunes Ribeiro, a Morfologia conversa com a Semântica Lexical. Seguindo o modelo de descrição de afixos por meio de um conjunto de traços semânticos, proposto por Rochelle Lieber em seu livro intitulado Morphology and Lexical Semantics, de 2004, os autores propõem que o significado de negação de estado associado a formações com o prefixo des- não é mais produtivo no PB atual, identificando, então, a prevalência do sentido de reversão de uma trajetória de mudança em novas palavras formadas com esse prefixo nessa língua. 
No que concerne à interface entre a Morfologia e a Sintaxe, Danniel Carvalho, em seu artigo intitulado $O$ traço de gênero na morfossintaxe do português, revisita as discussões acerca do estatuto do traço de gênero na teoria gramatical. $\mathrm{O}$ autor propõe que esse traço participa de relações de concordância que são estabelecidas na computação sintática (Chomsky 2000, 2001, Carstens 2000, 2010), levantando, então, a hipótese de que tal traço seja tratado como um elemento efetivamente sintático, ao invés de ser compreendido como algo relevante somente no nível das interfaces.

$\mathrm{O}$ artigo intitulado A relação entre a presença do núcleo Voice e o clítico SE no português brasileiro, de Janayna Carvalho, também discute a questão da interface entre a Morfologia e a Sintaxe através do estudo de construções anticausativas, médias e impessoais do $\mathrm{PB}$, que revelam processos de mudança sintática que ocorreram ou estão ocorrendo na língua. A autora observa que, apesar de serem comuns sem o clítico SE nos dias atuais, construções como essas eram morfologicamente marcadas por esse clítico em sincronias anteriores. Em sua análise, que tem o modelo da Morfologia Distribuída como fundamentação teórica, as mudanças nas estruturas dos eventos denotados por essas construções possuem uma origem em comum, nomeadamente, a perda de SE que nucleia um tipo específico de projeção Voice.

As relações entre Morfologia e Estrutura Argumental também são investigadas no trabalho intitulado Sincretismos morfológicos nas construções reflexivas e anticausativas em kadiwéu, de João Paulo Lazzarini Cyrino, que aborda o estatuto dos morfemas $d$ :- e $n$ - na língua indígena brasileira kadiwéu. Esses morfemas co-ocorrem em construções reflexivas e anticausativas, apesar de também ocorrerem independentemente e de estarem associados a diferentes fenômenos: $n$ - é marca de construção antipassiva e $d$ :- ocorre na presença de argumentos internos em posição pré-verbal. O modelo da Morfologia Distribuída e a proposta de Alboiu, Barrie e Frigeni (2004) para os reflexivos sugere a análise de $n$ - como marca passiva (Baker; Johnson; Roberts 1989), enquanto $d$ :- resulta de fissão no nó de concordância, desencadeada pela concordância com argumento no caso absolutivo.

A discussão presente no artigo intitulado $A$ morfossintaxe de compostos $X-V$ em inglês, de Ana Paula Scher, remete à questão dos tipos de processos morfológicos disponíveis nas línguas naturais: concatenativos e não concatenativos (Bye; Svenonious 2012). De modo mais particular, a autora descreve algumas das propriedades sintático-semânticas de compostos verbais na língua inglesa, tais como babysit, dry-clean e self-destruct, muitas vezes tratados como casos de derivação regressiva, ou seja, como resultantes de um processo morfológico de natureza não concatenativa. Sua proposta é a de que dados como esses não só podem ser tratados como resultantes de processos concatenativos, mas, também podem receber uma análise estrutural baseada nos pressupostos do modelo da Morfologia Distribuída em detrimento de análises lexicalistas. 
Um modelo otimalista de análise linguística, mais especificamente rotulado como HS-OI (McCarthy 2011), é utilizado no artigo intitulado Exponência de gênero e classe temática em português brasileiro, de Luiz Carlos Schwindt. Dentro de uma perspectiva de base realizacional, o trabalho estabelece uma hierarquia entre as marcas de gênero e de classe temática no PB. Mais especificamente, a hierarquia resulta em uma preferência da realização do expoente fonológico de classe sobre o de gênero, no caso do masculino, mas do expoente fonológico de gênero sobre o de classe, no caso do feminino.

Finalmente, no artigo intitulado $O$ status morfoprosódico dos sufixos -inho/-zinho, -mente e -íssimo no Português Brasileiro, Camila Witt Ulrich e Luiz Carlos Schwindt apresentam um exercício teórico-experimental de descrição e análise de palavras com os sufixos -inho/-zinho, -mente e -íssimo. Diferentemente de outros afixos, os autores propõem que esses se anexam a palavras morfológicas (locus morfológico) e a palavras prosódicas (locus prosódico), pois não se submetem a processos que acontecem no nível da raiz e não se submetem a processos que acontecem no nível da palavra prosódica.

\section{Considerações Finais}

Este artigo introdutório cumpre duas funções gerais. A primeira delas é a de registrar a história da constituição do CBM, destacando cada uma das suas edições até então. A segunda delas, por sua vez, é oferecer ao leitor um panorama dos artigos contidos neste volume da Revista D.E.L.T.A., que reúne trabalhos apresentados na terceira edição do evento em questão.

Para terminar essas palavras de apresentação deste volume da DELTA, gostaríamos de formalizar alguns agradecimentos importantes. Primeiramente, agradecemos aos autores dos trabalhos aqui publicados por nos confiarem a tarefa de editoração deste volume com os trabalhos apresentados no III CBM. É fundamental agradecer, também, aos editores da revista, professores Tony Berber Sardinha, Mary Kato e Maria Aparecida Caltabiano, pelo suporte constante durante os trabalhos de preparo dos manuscritos. Finalmente, agradecemos ao Departamento de Linguística da USP pelo apoio para a realização do III CBM e para a produção efetiva deste volume da revista.

Esperamos que as ideias defendidas nos trabalhos aqui publicados possam continuar inspirando outras pesquisas e, assim, ajudar a promover a ampliação do debate sobre as questões morfológicas.

Recebido em: 07/07/2017 Aprovado em: 07/07/2017

Emails:

Ana Paula Scher anascher@usp.br Indaiá Bassani indaia.bassani@unifesp.br Paula Roberta Gabbai Armelin paula.rg.armelin@gmail.com 


\section{Referências}

ALBOIU, Gabriela; BARRIE, Michael; FRIGENI, Chiara. 2004. SE and the Unnacusative-Unergative Paradox. In: COENE, Martine; De CUYPER, Gretel; D’HULST, Yves (Eds.) Antwerp Papers in Linguistics 107. Universiteit Antwerp.

BAKER, Mark; BOBALJIK, Jonathan. 2015. On Inherent and Dependent Theories of Case. Ms.

BORER, Hagit. 2005a. In Name Only: Structuring Sense, Vol. I. Oxford: Oxford University, Press.

BORER, Hagit. 2005b. The Normal Course of Events: Structuring Sense, Vol. II. Oxford:Oxford University Press.

BORER, Hagit. 2013. Taking Form: Structuring Sense, Vol. III. Oxford: Oxford University Press.

CARSTENS, Vicki. 2010. Implications of grammatical gender for the theory of uninterpretable features. In PUTNAM, Michael (Ed.) Exploring Crash-Proof Grammars. Amsterdam: Benjamins: 31-57.

CARSTENS, Vicki. 2000. Concord in Minimalist Theory. Linguistic Inquiry, 31: 319-355.

BYE, Patrick; SVENONIOUS, Peter. 2012. Non-concatenative morphology as epiphenomenon. In TROMMER, J. (Ed.) The Morphology and Phonology of Exponence, Oxford: Oxford University Press, p. 427-495.

HALLE, Morris; MARANTZ, Alec. 1993. Distributed Morphology and the pieces of inflection. In: HALE, Ken; KEYSER, Samuel Jay (Eds.). The view from Building 20. Cambridge, MA: MIT Press, p. 111-176.

LIEBER, Rochelle. Morphology and Lexical Semantics. Cambridge: Cambridge University Press, 2004.

MARANTZ, Alec. 1997. No Escape from Syntax: Don’t try Morphological Analysis in the privacy of your own Lexicon. In: DIMITRIADIS, Alexis; SIEGEL, Laura; SUREK-CLARK, Clarissa; WILLIAMS, Alexander (Eds.). Proceedings of the $21^{\text {st }}$ Penn Linguistics Colloquium. Philadelphia: UPenn Working Papers in Linguistics, p. 201-225.

MCCARTHY, John J. 2011. Pausal phonology and morpheme realization. In: BOROWSKY, Tony; KAWAHARA, Shigeto; SHINYA, Takahito, SUGAHARA, Mariko (Eds.). Prosody Matters: Essays in Honor of Lisa Selkirk. London: Equinox.

TALMY, Leonard. 2000. Toward a cognitive semantics. Cambridge: The MIT Press.

TALMY, Leonard. 2009. Main Verb Properties and Equipollent Framing. In: GUO; Jian-Sheng; LIEVEN, Elena; BUDWIG, Nancy; ERVIN-TRIPP, Susan; NAKAMURA, Keiko; ÖZÇALIŞKAN, Şeyda (Eds.). Crosslinguistic Approaches to the Psychology of Language: Research in the Tradition of Dan Isaac Slobin. Mahwah: Lawrence Erlbaum Associates.

TALMY, Leonard. 2012. Main verb properties. International Journal of Cognitive Linguistics 3: 1-24. 